Review Article

\title{
MMP Inhibitors and Dentin Bonding: Systematic Review and Meta-Analysis
}

\author{
O. Kiuru, ${ }^{1}$ J. Sinervo, ${ }^{1}$ H. Vähänikkilä, ${ }^{2}$ V. Anttonen $\mathbb{D}^{1,3}$ and L. Tjäderhane $\mathbb{D}^{1,3,4}$ \\ ${ }^{1}$ Research Unit of Oral Health Sciences, Department of Cariology,Endodontology and Paediatric Dentistry, University of Oulu, \\ Oulu, Finland \\ ${ }^{2}$ Infrastructure of Population Studies, Faculty of Medicine, University of Oulu, Oulu, Finland \\ ${ }^{3}$ Medical Research Centre, University of Oulu and Oulu University Hospital, Oulu, Finland \\ ${ }^{4}$ Department of Oral and Maxillofacial Diseases, University of Helsinki, and Helsinki University Hospital, Helsinki, Finland
}

Correspondence should be addressed to L. Tjäderhane; 1.tjaderhane@helsinki.fi

Received 2 April 2021; Accepted 19 May 2021; Published 29 May 2021

Academic Editor: Gaetano Isola

Copyright (C) 2021 O. Kiuru et al. This is an open access article distributed under the Creative Commons Attribution License, which permits unrestricted use, distribution, and reproduction in any medium, provided the original work is properly cited.

Objectives. Resin-dentin bond strength decreases over time. This reduction is related to the loss of hybrid layer integrity. Collagenolytic enzymes, especially matrix metalloproteinases (MMPs), are responsible for the degradation of the collagen matrix of the hybrid layer. Various MMP inhibitors with the ability to prevent enzymatic degradation have been identified. This study aimed to systematically review the literature for studies which evaluated the effect of MMP inhibitors on the immediate and aged dentin bond strengths. Study SelectionScreening and analysis were carried out by two reviewers. Two databases were searched, and from a total of 740 articles, 43 were accepted for full review. 21 articles with $0.2 \%-2 \%$ chlorhexidine (CHX) treatments were included for meta-analysis. A risk of bias assessment was performed on all studies chosen for meta-analysis. A variety of MMP inhibitors have been studied, CHX being the most widely used. Conclusions. A clear trend for a lower loss of dentin bond strength was observed with different MMP inhibitors. In meta-analysis, no significant difference was seen between the CHX and control in the immediate bond strengths. Bond strengths in the CHX group were significantly higher than the control group after aging $(P<0.001)$. The percentage of fractures occurring at the adhesive interface increased after aging. Five out of 21 studies included in the meta-analysis had high and the rest medium risk of bias. More long-term studies with lower risks of bias should be carried out to increase the reliability of results. Clinical RelevanceThe use of MMP inhibition with chlorhexidine can be recommended to increase the longevity of resin-dentin bond strength.

\section{Introduction}

Studies have shown that the bond between the adhesive systems and dentin weakens over time. This decrease in bond strength is related to the degradation of the hybrid layer $[1,2]$. The hybrid layer is the area of adhesion formed by the dentin collagen matrix and resin adhesive. After exposure to acid (etch-and-rinse adhesives) or acidic monomers (self-etch adhesives), the demineralized dentin collagen matrix is infiltrated with the applied adhesive resin $[3,4]$. The collagen matrix is vulnerable to enzymatic degradation by the endogenous collagenolytic enzymes, matrix metalloproteinases (MMPs) and cysteine cathepsins, and these enzymes play an important role in bond destruction [2]. MMP inhibitors can prevent the function of these enzymes, with chlorhexidine (CHX), galardin, and benzalkonium chloride being the most widely studied $[5,6]$. More recently, collagen cross-linker agents have also been shown to inhibit protease enzymes [7].

Systematic review and meta-analysis by Montagner et al. [8] indicated that CHX is effective in maintaining long-term bond strength. This study aimed to provide an updated systematic review and meta-analysis of the literature involving studies which evaluated the effect of MMP inhibitors on the immediate and aged resin-dentin bond strength. The hypothesis was that MMP inhibitors have a significant effect on the longevity of bond strength, which becomes evident after 6 months. 


\section{Materials and Methods}

In this systematic literature review, two separate electronic databases were used (PubMed and Scopus). Suitable search phrases for both databases were constructed using the following search phrases as a guideline: (matrix metalloproteinase* OR MMPs inhibitor* OR protease inhibitor* OR chlorhexidine $*$ OR benzalkonium chloride $*$ OR BAC $*$ ) AND (dentin $*$ adhesive $*$ OR adhesive system $*$ OR hybrid layer* OR bond $*$ ) AND (ag* OR stability* OR durability* OR strength $*$ OR long-term $*$ ) and "cross-linker OR crosslinker": e.g., with the following search phrases "grape seed extract AND dentin AND bonding" or "proanthocyanidin AND dentin AND bonding."

The search phrases for each database were altered and optimized so that the resulting articles were as relevant as possible to the targeted articles. The final search phrases used were as follows:

PubMed: $((()((()(($ proanthocyanidin AND dentin AND bonding) OR (grape seed extract AND dentin AND bonding) OR chlorhexidine* [Text Word] OR "benzalkonium chloride*" [Text Word] OR BAC* [Text Word] OR "matrix metalloproteinase inhibitor*" [Text Word] OR "MMP* inhibitor*" [Text Word] OR "protease inhibitor*" [Text Word] $)))$ AND ((ag* [Text Word] OR stability* [Text Word] OR durab $*$ [Text Word] OR strength $*$ [Text Word] OR long-term* [Text Word]))) AND ((dentin* AND adhesive $*$ [Text Word] OR adhesive system $*$ " [Text Word] OR "hybrid layer*" [Text Word] OR bond* [Text Word])))) OR ((“"matrix metalloproteinase*" [Text Word]) AND ((ag* [Text Word] OR stability* [Text Word] OR durab* [Text Word] OR strength* [Text Word] OR long-term* [Text Word])) ) AND ((dentin* AND adhesive* [Text Word] OR "adhesive system*" [Text Word] OR "hybrid layer*" [Text Word] OR bond* [Text Word]))))) OR (()( ("matrix metalloproteinase inhibitor*” [Text Word] OR "MMP* inhibitor*" [Text Word])) AND bond strength [Text Word])) OR (("Matrix Metalloproteinase Inhibitors”[Mesh]) AND ((“"Dental Bonding”[Mesh: noexp]) OR "Light-Curing of Dental Adhesives"[Mesh]) OR "SelfCuring of Dental Resins"[Mesh])))).

Scopus: (TITLE-ABS-KEY ((proanthocyanidin AND dentin AND bonding) OR (grape seed extract AND dentin AND bonding) OR "matrix metalloproteinase*" OR "MMP* inhibitor*" OR "protease inhibitor*" OR chlorhexidine* OR "benzalkonium chloride*" OR bac*) AND TITLE-ABS-KEY (adhe* OR "hybrid layer*" OR bond*) AND TITLE-ABS-KEY (ag* OR stability* OR durab $*$ OR strength $*$ OR long-term $*$ ) AND TITLE-ABSKEY (dentin*)) AND NOT INDEX (medline) AND (LIMIT-TO (SUBJAREA, “DENT”)).

Using these search phrases, 531 articles were found on PubMed and 209 articles on Scopus. The search included all articles published before 5.7.2018. After the database search, screening was performed by two individuals on all articles to single out the relevant ones. For the screening, the following predetermined rejection criteria were used: under 6 months aging (follow-up), thermocycling used for aging, no measured data of bond strength, no MMP inhibitors used during bonding, no control group, review articles, or other interests, e.g., root canal sealers and root canal posts. If any one of these factors was present, the article was rejected. The article also had to be written in English.

The screening was performed in three stages (Figure 1). During the first stage, only the title and the abstract of the article were used to determine whether any of the rejection criteria was present. The initial screening resulted in 126 articles from PubMed and 22 articles from Scopus. During the second stage, the complete text of all remaining articles was read and interpreted by the individual screeners (a total of 148). The same rejection criteria were used, and after careful selection, 59 articles from PubMed and seven articles from Scopus were accepted $(n=66)$. A final screening was performed with both reviewers present. Five of the seven articles found in Scopus were the same as on PubMed. Furthermore, 18 studies were rejected due to the rejection criteria and lack of available data, leaving 43 accepted articles. In addition, studies done on carious teeth were excluded.

Due to the wide heterogeneity between the studies using other MMP inhibitors than CHX, only data involving $0.2-2 \%$ CHX would be used for the meta-analysis. The 21 chosen articles were allocated into six different comparison groups depending on whether the samples had been aged for 6,12 , or 24 months and whether an etch-and-rinse or selfetching system had been used. No articles were found for the 12-month storage time and self-etch, so this group was excluded. The bond strength, sample size $(N)$, and standard deviation data for the respective storage times and controls were retrieved from the articles. The $N$ for each group represented the total number of teeth used in each comparison group. Pooled effect estimates were attained by comparing the means of each bond strength value, expressed as the raw mean difference among the groups. Statistical heterogeneity of the treatment effect was assessed via the Cochran $Q$ test, with $P<0.05$ considered significant, and the inconsistency $I^{2}$ test, in which values $>50 \%$ were considered to indicate high heterogeneity. Meta-analysis on the chosen comparison groups was carried out using the MedCalc (version 19.2.1: MedCalc Software Ltd., Ostend, Belgium).

2.1. Assessment of Risk of Bias. The risk of bias evaluation, adapted from a previous study [8], evaluated the following parameters for the study's quality assessment: randomization, use of intact teeth, use of materials according to the instructions, adhesive procedures performed by the same operator, description of sample size calculation, and blinding of testing. The articles reporting 5 to 6 items were classified as low risk of bias, 3 or 4 as medium risk, and only 1 or 2 as high risk.

\section{Results}

From the initial 740 articles, 43 articles with altogether 240 groups were subjected to a comprehensive examination (Table 1). Altogether, 21 different enzyme inhibitors were tested. The most commonly used MMP inhibitor was CHX 


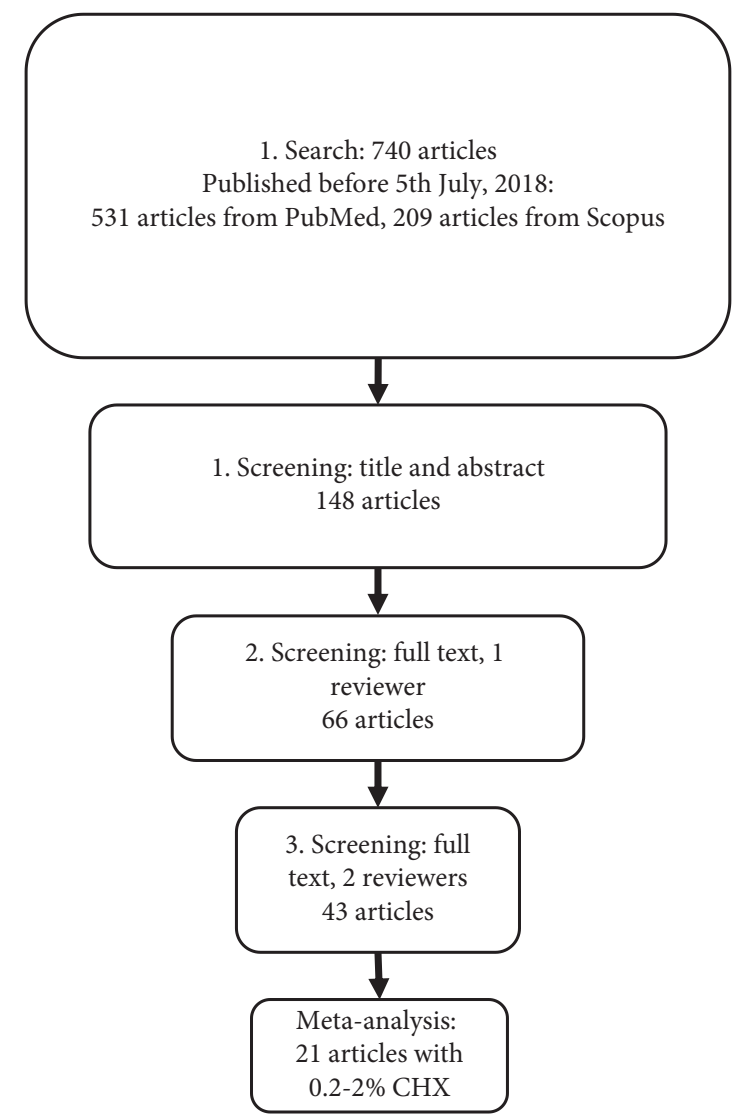

Figure 1: Protocol for the systematic literature review.

(32 studies), followed by BAC (seven studies). Artificial saliva and distilled water were the most commonly used modes of storage for aging. Percentage decreases in bond strength were calculated, and a general trend in the decrease of bond strength with time could be observed. A clear trend for the lower decrease in bond strength with MMP inhibitors in 35 out of 43 studies was also observed, with 13 different enzyme inhibitors showing significantly (at least 50\%) lower percentage loss of bond strength compared to the respective control group. The adhesive-mixed fracture percentages for the control and CHX groups ranged from 0 to 100 at baseline, from 41 to 100 at 6 months, from 50 to 100 for the control, and from 55 to 100 for CHX at 12 months, and from 77 to 100 for the control and from 75 to 100 for CHX in the final aging group of 24 months.

3.1. Meta-Analysis. A total of 21 articles were subjected to meta-analysis. The first analysis involved CHX vs. control at baseline (Figure 2), including 37 data sets from 21 articles. No significant difference in bond strength was present between the groups $(P=0.308)$. The heterogeneity between the studies was low (Cochran's $Q P>0.05, I^{2} 21.7 \%$ ).

Thirty data sets from 16 articles were available for the comparison between $\mathrm{CHX}$ vs. control after 6 months aging (Figure 3(a)). Bond strengths in the $\mathrm{CHX}$ group were significantly higher than in the control group after aging $(P<0.001)$. The heterogeneity between the studies was high (Cochran's $Q P<0.05, I^{2} 82.1 \%$ ).
To compare CHX vs. control after 12 months aging, 17 data sets from 10 articles were available (Figure 3(b)). Bond strengths in the CHX group were significantly higher than in the control group $(P<0.001)$. The heterogeneity between the studies was high (Cochran's $Q P<0.05, I^{2} 75.0 \%$ ).

The last analysis involved CHX vs. control after 24 months aging, including six data sets from three articles (Figure 3(c)). Bond strengths in the CHX group were significantly higher than in the control group $(P<0.001)$. The heterogeneity between the studies was extremely low (Cochran's $Q P=0.817, I^{2} 0 \%$ ).

3.2. Risk of Bias. Of the 21 articles selected for the metaanalysis, five were classified as having a high risk and 16 as a medium risk of bias. None of the articles had a low risk of bias (Table 2).

\section{Discussion}

The general trend of all 43 articles with 21 different collagenolytic enzyme inhibitor protocols demonstrated markedly lower loss of bond strength with enzyme inhibition. Studies involving other MMP inhibitors were excluded from the meta-analysis due to a wide range of heterogeneity in the inhibitors used and a small number of studies for each inhibitor except for CHX. All three comparisons between the $\mathrm{CHX}$-treated and controls after aging for at least six months demonstrated significantly higher bond strength with $\mathrm{CHX}$. Thus, the hypothesis was accepted.

The results of the meta-analysis showed that the use of CHX has no significant effect on immediate resin-dentin bond strength. The finding is in line with a previous study [8]. Two studies $[14,23]$ have shown a significant decrease in the immediate bond strength of the CHX group compared to the control. After reviewing the articles, no clear explanation for this difference could be identified, although several differences in the application of CHX were noted. Giacomini and co-authors speculated that the use of acidic CHX after acid etching may have resulted in increased collagen exposure, possibly reducing the immediate bond strength [14].

After aging for 6, 12, and 24 months, the meta-analysis demonstrated significantly better bond strength with CHX compared to the control groups. Despite the heterogeneity of the studies, 6- and 12-month analyses indicate the advantage of using CHX to preserve the bond strength. In addition, with the studies evaluating the bond strength after 24 months of aging, the homogeneity of the data was striking $\left(I^{2}\right.$ $0 \%)$. Indeed, longer aging seems to increase the difference between the bond strengths of CHX and control groups.

The immediate fracture percentage at the adhesive interface was practically the same for MMP inhibitor and control groups, although some isolated differences can be identified. It can generally be observed that as the follow-up time increases, the percentage of fractures occurring at the adhesive interface seems to increase, regardless of enzyme inhibition. This may be due to slow degradation of hybrid layer collagen despite the enzyme inhibition, the hydrolytic degradation of the resin component, or-most likely-to 
TABLE 1: Percentages of reduction in bond strength during the follow-up period.

\begin{tabular}{|c|c|c|c|c|c|c|}
\hline \multirow[t]{2}{*}{ Article } & \multirow{2}{*}{$\begin{array}{l}\text { Adhesive/mixed failure modes (\%) in } \\
\text { groups immediately/after aging }\end{array}$} & \multirow[t]{2}{*}{$N$} & \multirow{2}{*}{$\begin{array}{l}\text { Technique of bonding }(\mathrm{E} \& \mathrm{R} / \\
\text { SE) MMP inhibitor }+\%\end{array}$} & \multicolumn{3}{|c|}{$\begin{array}{c}\text { Bond strength reduction (\%) } \\
\text { after aging }\end{array}$} \\
\hline & & & & $6 \mathrm{~m}$ & $12 \mathrm{~m}$ & $>12 \mathrm{~m}$ \\
\hline \multirow{5}{*}{ Li et al. [9] } & \multirow{5}{*}{ NA } & \multirow{5}{*}{ NA } & $\mathrm{E} \& \mathrm{R}$ & & & \\
\hline & & & DMSO $1 \%$ & $17.4^{*}$ & & \\
\hline & & & GD 5\% & $11.9^{*}$ & & \\
\hline & & & BAI $2.5 \mu \mathrm{g} / \mathrm{mL}$ & $10.1^{*}$ & & \\
\hline & & & Control & 36.0 & & \\
\hline \multirow{12}{*}{ Malaquias et al. [10] } & $\begin{array}{c}\text { Group: immediate } / 24 \mathrm{~m} \\
\text { Ambar }^{\circledR}\end{array}$ & \multirow{12}{*}{50} & $\mathrm{E} \& \mathrm{R}$ & & & $24 \mathrm{~m}$ \\
\hline & CHX 0.01\%: 79.2/84.3 & & CHX 0.01\% & & & $16.7^{*}$ \\
\hline & CHX 0.05\%: 81.3/88.1 & & CHX 0.05\% & & & $17.0^{*}$ \\
\hline & CHX 0.1\%: 80.5/86.9 & & CHX $0.1 \%$ & & & $10.0^{*}$ \\
\hline & CHX 0.2\%: $72.7 / 76.8$ & & CHX $0.2 \%$ & & & $10.4^{*}$ \\
\hline & Control: $81 / 83.4$ & & Control & & & 40.2 \\
\hline & $\begin{array}{c}\text { Group: immediate } / 24 \mathrm{~m} \\
\text { XP-Bond }{ }^{\circledR}\end{array}$ & & $\mathrm{E} \& \mathrm{R}$ & & & \\
\hline & CHX 0.01\%: 75.7/77.7 & & CHX $0.01 \%$ & & & $33.8^{*}$ \\
\hline & CHX 0.05\%: 81/78.4 & & CHX $0.05 \%$ & & & $32.1^{*}$ \\
\hline & CHX 0.1\%: 69.8/94.8 & & CHX $0.1 \%$ & & & $29.1^{*}$ \\
\hline & CHX 0.2\%: 74/82.1 & & CHX $0.2 \%$ & & & $29.3^{*}$ \\
\hline & Control: 81.6/77.2 & & Control & & & 53.3 \\
\hline \multirow{4}{*}{ Ou et al. [11] } & Group: immediate $/ 6 \mathrm{~m} / 12 \mathrm{~m}$ & \multirow{4}{*}{60} & E\&R & & & \\
\hline & MMP8-I/90/70/75 & & MMP8-I & $1.1^{*}$ & $1.8^{*}$ & \\
\hline & CHX 2\%: 100/95/95 & & CHX $2 \%$ & 0.7 & $5.3^{*}$ & \\
\hline & Control: 95/80/90 & & Control & 17.2 & 24.1 & \\
\hline \multirow{8}{*}{ El Gezawi et al. [12] } & $\begin{array}{c}\text { Group: immediate } / 6 \mathrm{~m} \\
\mu \mathrm{TBS}\end{array}$ & \multirow{8}{*}{48} & & & & \\
\hline & MDPB: 68/74 & & MDPB-SE & 30.2 & & \\
\hline & BAC: $68 / 72$ & & BAC-PA & 58.5 & & \\
\hline & Control: 72/78 & & Control & 64.3 & & \\
\hline & 4-point loading & & & & & \\
\hline & MDPB: $68 / 70^{\circ}$ & & MDPB-SE & $31.6^{*}$ & & \\
\hline & BAC: $72 / 86$ & & BAC-PA & 62.9 & & \\
\hline & Control: $78 / 84$ & & Control & 69.1 & & \\
\hline \multirow{3}{*}{ Maravic et al. [13] } & Group: immediate/12 m & \multirow{3}{*}{36} & $\mathrm{E} \& \mathrm{R}$ & & & \\
\hline & ACR: $65 / 90$ & & ACR $0.01 \%$ & & $14.4^{*}$ & \\
\hline & Control: $67 / 82$ & & Control & & 46.1 & \\
\hline \multirow{11}{*}{ Giacomini et al. [14] } & $\begin{array}{l}\text { Group: immediate } / 6 \mathrm{~m} \\
\text { Normal dentin }\end{array}$ & \multirow{11}{*}{90} & $\mathrm{E} \& \mathrm{R}$ & & & \\
\hline & CHX 2\%: $100 / 100$ & & CHX 2\% & $41.8^{*}$ & & \\
\hline & E-64: $96 / 96$ & & E-64 $5 \mu \mathrm{m}$ & $26.6^{*}$ & & \\
\hline & Control: $96 / 92$ & & Control & 22.3 & & \\
\hline & Artificially carious dentin & & & & & \\
\hline & CHX 2\%: $100 / 100$ & & CHX 2\% & 9.9 & & \\
\hline & E-64: 96/96 & & E-64. $5 \mu \mathrm{m}$ & 15.1 & & \\
\hline & $\begin{array}{l}\text { Control: 100/100 } \\
\text { Eroded dentin }\end{array}$ & & Control & 13.4 & & \\
\hline & CHX 2\%: 100/100 & & CHX 2\% & $10.7^{*}$ & & \\
\hline & E-64: $100 / 100$ & & E-64 $5 \mu \mathrm{m}$ & 8.4 & & \\
\hline & Control/96/100 & & Control & 12.7 & & \\
\hline
\end{tabular}


TABle 1: Continued.

\begin{tabular}{|c|c|c|c|c|c|c|}
\hline \multirow[t]{2}{*}{ Article } & \multirow{2}{*}{$\begin{array}{l}\text { Adhesive/mixed failure modes (\%) in } \\
\text { groups immediately/after aging }\end{array}$} & \multirow[t]{2}{*}{$N$} & \multirow{2}{*}{$\begin{array}{l}\text { Technique of bonding (E\&R/ } \\
\text { SE) MMP inhibitor }+\%\end{array}$} & \multicolumn{3}{|c|}{$\begin{array}{c}\text { Bond strength reduction (\%) } \\
\text { after aging }\end{array}$} \\
\hline & & & & $6 \mathrm{~m}$ & $12 \mathrm{~m}$ & $>12 \mathrm{~m}$ \\
\hline \multirow{12}{*}{ Daood et al. [15] } & $\begin{array}{l}\text { Group: immediate } / 12 \mathrm{~m} \\
\text { Adper }^{\mathrm{TM}} \text { Single Bond } 2\end{array}$ & \multirow{12}{*}{60} & $E \& R$ & & & \\
\hline & CHX 2\%: 64/72 & & CHX $2 \%$ & $4^{*}$ & $8^{*}$ & \\
\hline & QAS $2 \%: 52 / 65$ & & QAS $2 \%$ & $-8.1^{*}$ & $-3.6^{*}$ & \\
\hline & QAS 5\%: 75/64 & & QAS 5\% & $4.2^{*}$ & $-6.0^{*}$ & \\
\hline & QAS $10 \%: 72 / 67$ & & QAS $10 \%$ & 10.5 & 23.1 & \\
\hline & Control: $63 / 66$ & & Control & 14.5 & 29.5 & \\
\hline & Prime \& Bond ${ }^{\circledR} \mathrm{NT}^{\mathrm{TM}}$ & & E\&R & & & \\
\hline & CHX 2\%: 80/55 & & CHX $2 \%$ & 3.3 & $8.8^{*}$ & \\
\hline & QAS 2\%: 59/64 & & QAS $2 \%$ & -2.2 & $-4.4^{*}$ & \\
\hline & QAS 5\%: 74/74 & & QAS 5\% & $5.6^{*}$ & $11.3^{*}$ & \\
\hline & QAS $10 \%: 75 / 79$ & & QAS10\% & $6.9^{*}$ & $13.8^{*}$ & \\
\hline & Control: 64/74 & & Control & 12.2 & 20.9 & \\
\hline \multirow{9}{*}{ Venigalla et al. [16] } & Group: immediate $/ 6 \mathrm{~m}$ & \multirow{9}{*}{80} & $E \& R$ & & & \\
\hline & RF WWB: $73 / 47$ & & RF $0.1 \%$ WWB & $2.5^{*}$ & & \\
\hline & CD WWB: $73 / 60$ & & $1 \mathrm{~m} \mathrm{CD} \mathrm{WWB}$ & $5.6^{*}$ & & \\
\hline & PAC WWP: $67 / 47$ & & $6.5 \%$ PAC WWB & $17^{*}$ & & \\
\hline & Control WWB: $87 / 60$ & & Control WWB & 24 & & \\
\hline & RF EWB: 53/47 & & RF $0.1 \%$ EWB & $0.6^{*}$ & & \\
\hline & CD EWB: $67 / 60$ & & $1 \mathrm{~m} \mathrm{CD} \mathrm{EWB}$ & $4.7^{*}$ & & \\
\hline & PAC EWB: $60 / 53$ & & $6.5 \%$ PAC EWB & $5.6^{*}$ & & \\
\hline & Control EWB: $67 / 47$ & & Control EWB & 10.2 & & \\
\hline \multirow{4}{*}{ Carvalho et al. [17] } & Group: immediate/6m & \multirow{4}{*}{30} & $\mathrm{E} \& \mathrm{R}$ & & & \\
\hline & Green tea: $36 / 68$ & & Green tea $2 \%$ & $-55.2^{*}$ & & \\
\hline & CHX 2\%: 66/78 & & CHX 2\% & 1.3 & & \\
\hline & Control: $81 / 68$ & & Control & 11.1 & & \\
\hline \multirow{4}{*}{ Barcellos et al. [18] } & \multirow{4}{*}{ NA } & \multirow{4}{*}{60} & $\mathrm{E} \& \mathrm{R}$ & & & \\
\hline & & & $\mathrm{ZnOn} 1$ wt.\% & $3.1^{*}$ & & \\
\hline & & & Zn-Mt, 1 wt.\% & 46.1 & & \\
\hline & & & Control & 44.4 & & \\
\hline \multirow{10}{*}{ Hass et al. [19] } & \multirow{10}{*}{ NA } & \multirow{10}{*}{40} & E\&R Single Bond Plus ${ }^{\circledR}$ & & & $18 \mathrm{~m}$ \\
\hline & & & PAC $6.5 w t \%$ & & & $11.9^{*}$ \\
\hline & & & UVA-RF $0.1 w t \%$ & & & $14.8^{*}$ \\
\hline & & & GD $5 w t \%$ & & & $22.9^{*}$ \\
\hline & & & Control & & & 64.8 \\
\hline & & & E\&R Tetric N-Bond ${ }^{\circledR}$ & & & \\
\hline & & & PAC $6.5 w t \%$ & & & $5.5^{*}$ \\
\hline & & & UVA-RF $0.1 w t \%$ & & & $20.3^{*}$ \\
\hline & & & GD $5 w t \%$ & & & $32.2^{*}$ \\
\hline & & & Control & & & 62.2 \\
\hline \multirow{8}{*}{ Loguercio et al. [20] } & $\begin{array}{l}\text { Group: immediate } / 24 \mathrm{~m} \\
\text { Prime \& Bond NT }{ }^{\circledR}\end{array}$ & \multirow{8}{*}{30} & $\mathrm{E} \& \mathrm{R}$ & & & $24 \mathrm{~m}$ \\
\hline & MC 2\%: $97 / 94$ & & MC $2 \%$ & & & $10.6^{*}$ \\
\hline & CHX 2\%: 85/94 & & $\mathrm{CHX} 2 \%$ & & & $17.9^{*}$ \\
\hline & Control: 80/78 & & Control & & & 44.2 \\
\hline & Adper ${ }^{\mathrm{TM}}$ Single Bond 2 & & E\&R & & & \\
\hline & MC 2\%: $98 / 96$ & & MC 2\% & & & $10.9^{*}$ \\
\hline & CHX 2\%: $93 / 90$ & & $\mathrm{CHX} 2 \%$ & & & $13.9^{*}$ \\
\hline & Control: 93/95 & & Control & & & 30.1 \\
\hline \multirow{3}{*}{ Hass et al. [21] } & Group: immediate/6 m & & $\mathrm{E} \& \mathrm{R}$ & & & \\
\hline & PAC 2\%-PA: 84/82 & 10 & PAC 2\%-PA & $-2.7^{*}$ & & \\
\hline & Control: 97/100 & & Control & 47.2 & & \\
\hline
\end{tabular}


TABle 1: Continued.

\begin{tabular}{|c|c|c|c|c|c|c|}
\hline \multirow[t]{2}{*}{ Article } & \multirow{2}{*}{$\begin{array}{l}\text { Adhesive/mixed failure modes (\%) in } \\
\text { groups immediately/after aging }\end{array}$} & \multirow[t]{2}{*}{$N$} & \multirow{2}{*}{$\begin{array}{l}\text { Technique of bonding (E\&R/ } \\
\text { SE) MMP inhibitor }+\%\end{array}$} & \multicolumn{3}{|c|}{$\begin{array}{c}\text { Bond strength reduction (\%) } \\
\text { after aging }\end{array}$} \\
\hline & & & & $6 \mathrm{~m}$ & $12 \mathrm{~m}$ & $>12 \mathrm{~m}$ \\
\hline \multirow{12}{*}{ Tekçe et al. [22] } & $\begin{array}{l}\text { Group: immediate } / 12 \mathrm{~m} \\
\text { SB Universal }{ }^{\circledR}\end{array}$ & \multirow{12}{*}{50} & & & & \\
\hline & BAC $1 \%: 58 / 75.5$ & & BAC $1 \%$ & & 23 & \\
\hline & CHX 2\%: 70.4/76 & & $\mathrm{CHX} 2 \%$ & & 8.9 & \\
\hline & EDTA $0.5 \mathrm{~m}: 66.1 / 73.4$ & & EDTA $0.5 \mathrm{~m}$ & & -0.9 & \\
\hline & SE: $60.6 / 78.3$ & & SE control & & 3.6 & \\
\hline & E\&R: $58.4 / 79$ & & E\&R control & & 13.1 & \\
\hline & All-Bond Universal ${ }^{\circledR}$ & & & & & \\
\hline & BAC 1\%: 70.2/71 & & BAC $1 \%$ & & 15.2 & \\
\hline & CHX 2\%: 60.5/73.5 & & CHX $2 \%$ & & 19.4 & \\
\hline & EDTA $0.5 \mathrm{~m} / 57 / 76$ & & EDTA $0.5 \mathrm{~m}$ & & 17.6 & \\
\hline & SE: $78.6 / 90$ & & SE control & & 21.6 & \\
\hline & E\&R: $65.5 / 71$ & & E\&R control & & 12.0 & \\
\hline \multirow{4}{*}{ Abu Nawareg et al. [23] } & Group: immediate $/ 6 \mathrm{~m} / 12 \mathrm{~m}$ & \multirow{4}{*}{36} & $\mathrm{E} \& \mathrm{R}$ & & & \\
\hline & CHX 2\%: 93.3/86.7/86.7 & & CHX $2 \%$ & $3.5^{*}$ & $5.9^{*}$ & \\
\hline & CHX-MA 2\%: 86.7/100/80 & & CHX-MA $2 \%$ & $-5.1^{*}$ & $-4.9^{*}$ & \\
\hline & Control: $93.3 / 100 / 86.7$ & & Control & 22.9 & 33.3 & \\
\hline \multirow{7}{*}{ da Silva et al. [24] } & $\begin{array}{l}\text { Group: immediate } / 6 \mathrm{~m} / 12 \mathrm{~m} \\
\text { Experimental adhesive }\end{array}$ & \multirow{7}{*}{36} & $E \& R$ & & & \\
\hline & GAL $5 \mu \mathrm{m}: 77 / 77 / 59$ & & GAL $5 \mu \mathrm{m}$ & 13.3 & 17.5 & \\
\hline & BAT $5 \mu \mathrm{m}: 71 / 71 / 84$ & & BAT $5 \mu \mathrm{m}$ & 10.9 & 15.3 & \\
\hline & GM1 $5 \mu \mathrm{m}: 72 / 72 / 57$ & & GM1 $5 \mu \mathrm{m}$ & 11.5 & 15.1 & \\
\hline & CHX 2\%: 70/72/63 & & CHX 2\% & 12.5 & 13.9 & \\
\hline & Control: 79/75/83 & & Control & 0.1 & 23.5 & \\
\hline & Single Bond 2: 68/74/84 & & Single Bond 2 & 5.7 & 20.3 & \\
\hline \multirow{4}{*}{ Montagner et al. [25] } & \multirow{4}{*}{ NA } & \multirow{4}{*}{36} & $E \& R$ & & & $18 \mathrm{~m}$ \\
\hline & & & $\mathrm{CHX} 2 \%$ & & & 20.6 \\
\hline & & & $\mathrm{NaOCl}$ & & & 25.7 \\
\hline & & & Control & & & 44.6 \\
\hline \multirow{6}{*}{ Sabatini et al. [26] } & \multirow{6}{*}{ NA } & \multirow{6}{*}{25} & $E \& R$ & & & \\
\hline & & & $\mathrm{CHX} 2 \%$ & & $1.3^{*}$ & \\
\hline & & & BAC-PA $1 \%$ & & 53.2 & \\
\hline & & & BAC $0.5 \%$ & & $9.1^{*}$ & \\
\hline & & & BAC 1\% & & $28.3^{*}$ & \\
\hline & & & Control & & 43.9 & \\
\hline \multirow{8}{*}{$\begin{array}{l}\text { Sabatini and Pashley } \\
{[27]}\end{array}$} & \multirow{8}{*}{ NA } & \multirow{8}{*}{35} & $\mathrm{E} \& \mathrm{R}$ & & & \\
\hline & & & $0.5 \% \mathrm{BAC}$ & $-2.6^{*}$ & $1.6^{*}$ & \\
\hline & & & $1.0 \% \mathrm{BAC}$ & $4.5^{*}$ & $-7.0^{*}$ & \\
\hline & & & $2.0 \% \mathrm{BAC}$ & $5.5^{*}$ & $13.4^{*}$ & \\
\hline & & & $0.5 \% \mathrm{MBAC}$ & $-11.2^{*}$ & $-26.4^{*}$ & \\
\hline & & & $1.0 \% \mathrm{MBAC}$ & $-5.4^{*}$ & $-23.1^{*}$ & \\
\hline & & & $2.0 \% \mathrm{MBAC}$ & $5.6^{*}$ & $1.0^{*}$ & \\
\hline & & & Control & 44.2 & 48.0 & \\
\hline \multirow{7}{*}{ André et al. [28] } & & & E\&R & & & \\
\hline & & & GD & & 5.8 & \\
\hline & & & GD-control & & $32.1^{*}$ & \\
\hline & NA & 60 & MDPB & & -8.9 & \\
\hline & & & MDPB-control & & 19.2 & \\
\hline & & & $0.2 \% \mathrm{CHX}$ & & 2.5 & \\
\hline & & & $0.2 \% \mathrm{CHX}$-control & & 13.5 & \\
\hline
\end{tabular}


TABle 1: Continued.

\begin{tabular}{|c|c|c|c|c|c|c|}
\hline \multirow[t]{2}{*}{ Article } & \multirow{2}{*}{$\begin{array}{l}\text { Adhesive/mixed failure modes (\%) in } \\
\text { groups immediately/after aging }\end{array}$} & \multirow[t]{2}{*}{$N$} & \multirow{2}{*}{$\begin{array}{l}\text { Technique of bonding }(\mathrm{E} \& \mathrm{R} / \\
\text { SE) MMP inhibitor }+\%\end{array}$} & \multicolumn{3}{|c|}{$\begin{array}{c}\text { Bond strength reduction (\%) } \\
\text { after aging }\end{array}$} \\
\hline & & & & $6 \mathrm{~m}$ & $12 \mathrm{~m}$ & $>12 \mathrm{~m}$ \\
\hline \multirow{9}{*}{ Manso et al. [29] } & $\begin{array}{l}\text { Group: immediate } / 6 \mathrm{~m} / 15 \mathrm{~m} \\
\text { All-Bond } 3^{\circledR}\end{array}$ & \multirow{9}{*}{48} & $E \& R$ & & & $15 \mathrm{~m}$ \\
\hline & CHX 1\% W: 73.5/51.6/58.3 & & CHX $1 \%$ water & -7.9 & & 1.9 \\
\hline & Control W: 50/44.5/50 & & Control water & -11.9 & & 7.4 \\
\hline & CHX 1\% E: 75/55.8/65.3 & & CHX $1 \%$ ethanol & 4.6 & & 27.6 \\
\hline & $\begin{array}{c}\text { Control E: } 56.8 / 62.7 / 54.1 \\
\text { Excite }^{\circledR}\end{array}$ & & $\begin{array}{c}\text { Control ethanol } \\
\text { E\&R }\end{array}$ & 5.1 & & 25.3 \\
\hline & CHX 1\% W: $77.7 / 73.8 / 76$ & & CHX $1 \%$ water & 8.2 & & -2.1 \\
\hline & Control W: 78/81.5/69.1 & & Control water & 15.0 & & 8.1 \\
\hline & CHX 1\% E: $62.1 / 47.2 / 59.2$ & & CHX $1 \%$ ethanol & -7.7 & & 7.2 \\
\hline & Control E: 56.7/41.2/76.6 & & Control ethanol & 10.3 & & 14.5 \\
\hline \multirow{10}{*}{ Ekambaram et al. [30] } & $\begin{array}{l}\text { Group: immediate/12 m } \\
\text { Sound dentin }\end{array}$ & \multirow{10}{*}{48} & E\&R & & & \\
\hline & EWB + CHX: 93.7/75 & & $\mathrm{EWB}+\mathrm{CHX} 2 \%$ & & $4.9^{*}$ & \\
\hline & EWB control: 100/68.8 & & EWB control & & $21.2^{*}$ & \\
\hline & WWB + CHX: 100/100 & & $\mathrm{WWB}+\mathrm{CHX}$ & & $0.3^{*}$ & \\
\hline & WWB control: 93.8/100 & & WWB control & & 27.6 & \\
\hline & Caries-affected dentin & & E\&R & & & \\
\hline & EWB + CHX: 68.8/81.2 & & $\mathrm{EWB}+\mathrm{CHX}$ & & $6.4^{*}$ & \\
\hline & EWB control: 75.5/62.6 & & EWB control & & $14.4^{*}$ & \\
\hline & WWB + CHX: 50/100 & & $\mathrm{WWB}+\mathrm{CHX}$ & & $18.7^{*}$ & \\
\hline & WWB control: $56.3 / 62.5$ & & WWB control & & 60.9 & \\
\hline \multirow{16}{*}{ Sabatini and Patel [31] } & $\begin{array}{l}\text { Group: immediate } / 6 \mathrm{~m} / 18 \mathrm{~m} \\
\text { OptiBond Solo Plus }{ }^{\circledR}\end{array}$ & \multirow{16}{*}{140} & $\mathrm{E} \& \mathrm{R}$ & & & $18 \mathrm{~m}$ \\
\hline & 2\% CHX: 70/70/60 & & $2 \% \mathrm{CHX}$ & 12.4 & & 6.0 \\
\hline & BAC-PA: $60 / 60 / 60$ & & BAC-PA & -4.7 & & -27.1 \\
\hline & $0.25 \%$ BAC: $60 / 70 / 60$ & & $0.25 \% \mathrm{BAC}$ & 32.4 & & 30.9 \\
\hline & $0.5 \%$ BAC: $70 / 70 / 80$ & & $0.5 \% \mathrm{BAC}$ & 1.8 & & $-95.0^{*}$ \\
\hline & $1.0 \%$ BAC: $70 / 70 / 100$ & & $1.0 \% \mathrm{BAC}$ & $-21.2^{*}$ & & $-46.3^{*}$ \\
\hline & $2.0 \%$ BAC: $80 / 70 / 70$ & & $2.0 \% \mathrm{BAC}$ & $15.7^{*}$ & & -19.1 \\
\hline & Control: 70/70/80 & & Control & -3.5 & & -1.9 \\
\hline & All-Bond $3^{\circledR}$ & & E\&R & & & $18 \mathrm{~m}$ \\
\hline & 2\% CHX: 80/70/70 & & $2 \% \mathrm{CHX}$ & -13.8 & & 15.5 \\
\hline & BAC-PA: 90/80/50 & & BAC-PA & -26.5 & & -9.6 \\
\hline & $0.25 \%$ BAC: $70 / 70 / 60$ & & $0.25 \% \mathrm{BAC}$ & 11.1 & & -33.3 \\
\hline & 0.5\% BAC: $70 / 60 / 70$ & & $0.5 \% \mathrm{BAC}$ & 9.8 & & -41.0 \\
\hline & $1.0 \%$ BAC: $90 / 80 / 90$ & & $1.0 \% \mathrm{BAC}$ & -0.5 & & -22.2 \\
\hline & $2.0 \%$ BAC: $90 / 80 / 70$ & & $2.0 \% \mathrm{BAC}$ & 10.7 & & -8.5 \\
\hline & Control: $80 / 70 / 70$ & & Control & -20.9 & & -15 \\
\hline \multirow{3}{*}{$\begin{array}{l}\text { Pomacóndor- } \\
\text { Hernández et al. [32] }\end{array}$} & \multirow{3}{*}{ NA } & \multirow{3}{*}{8} & SE & & & \\
\hline & & & CHX $2 \%$ & -8.1 & & \\
\hline & & & Control & 2.7 & & \\
\hline \multirow{8}{*}{ Verma et al. [33] } & \multirow{8}{*}{ NA } & \multirow{8}{*}{120} & E\&R Solobond $\mathrm{M}^{\circledR}$ & & & \\
\hline & & & CHX 2\% & $-8.6^{*}$ & & \\
\hline & & & PAC $30 \%$ & $6.8^{*}$ & & \\
\hline & & & Control & 45.1 & & \\
\hline & & & Tetric N Bond ${ }^{\circledR}$ & & & \\
\hline & & & $\mathrm{CHX} 2 \%$ & $0.9^{*}$ & & \\
\hline & & & PAC $30 \%$ & $0.7^{*}$ & & \\
\hline & & & Control & 36.2 & & \\
\hline \multirow{4}{*}{ Tjäderhane et al. [34] } & \multirow{4}{*}{ NA } & \multirow{4}{*}{20} & DMSO $0.5 \mathrm{~mm}$ & $-15.7^{*}$ & $-36.4^{*}$ & \\
\hline & & & Control & 37.2 & 30.4 & \\
\hline & & & DMSO $0.5 \mathrm{~mm}$ & $-12^{*}$ & $-6.6^{*}$ & \\
\hline & & & Control & 22.2 & 42.0 & \\
\hline
\end{tabular}


Table 1: Continued.

\begin{tabular}{|c|c|c|c|c|c|c|}
\hline \multirow[t]{2}{*}{ Article } & \multirow{2}{*}{$\begin{array}{l}\text { Adhesive/mixed failure modes (\%) in } \\
\text { groups immediately/after aging }\end{array}$} & \multirow[t]{2}{*}{$N$} & \multirow{2}{*}{$\begin{array}{l}\text { Technique of bonding (E\&R/ } \\
\text { SE) MMP inhibitor }+\%\end{array}$} & \multicolumn{3}{|c|}{$\begin{array}{l}\text { Bond strength reduction (\%) } \\
\text { after aging }\end{array}$} \\
\hline & & & & $6 \mathrm{~m}$ & $12 \mathrm{~m}$ & $>12 \mathrm{~m}$ \\
\hline \multirow{6}{*}{ Sabatini et al. [35] } & Group: immediate $/ 6 \mathrm{~m}$ & \multirow{6}{*}{25} & $\mathrm{E} \& \mathrm{R}$ & & & \\
\hline & CHX 2\%: 70/60 & & CHX $2 \%$ & $10.4^{*}$ & & \\
\hline & BAC-PA: $80 / 80$ & & BAC $1 \%$ PA & $18.4^{*}$ & & \\
\hline & BAC $0.5 \%: 70 / 80$ & & BAC $0.5 \%$-adhesive & $-0.5^{*}$ & & \\
\hline & BAC 01\%: $80 / 70$ & & BAC 1\%-adhesive & $-4.9^{*}$ & & \\
\hline & Control: 70/60 & & Control & 20.1 & & \\
\hline \multirow{5}{*}{ Simoes et al. [36] } & \multirow{5}{*}{ NA } & \multirow{5}{*}{36} & $\mathrm{E} \& \mathrm{R}$ & & & \\
\hline & & & $\mathrm{CHX}$ & 28.5 & & \\
\hline & & & Control & 32.7 & & \\
\hline & & & $\mathrm{CHX}+$ ethanol & 21.4 & & \\
\hline & & & Ethanol control & 7.6 & & \\
\hline \multirow{7}{*}{ Sabatini [37] } & Group: immediate $/ 6 \mathrm{~m}$ & \multirow{7}{*}{120} & $\mathrm{E} \& \mathrm{R}$ & & & \\
\hline & CHX $2 \%+0.2 \%$ CHX-adhesive: $70 / 50$ & & CHX $2 \%+0.2 \% \mathrm{CHX}$-adhesive & -4.2 & & \\
\hline & $0.2 \%$-adhesive: $80 / 60$ & & $0.2 \% \mathrm{CHX}$-adhesive & 5.2 & & \\
\hline & Control: $90 / 70$ & & Control & -14.3 & & \\
\hline & CHX $2 \%+0.2 \%$ CHX-adhesive: $60 / 60$ & & $\begin{array}{c}\text { SE } \\
\text { CHX } 2 \%+0.2 \% \text { CHX-adhesive }\end{array}$ & -13.8 & & \\
\hline & $0.2 \%$ CHX-adhesive: $50 / 80$ & & $0.2 \% \mathrm{CHX}$-adhesive & -17.4 & & \\
\hline & Control: $60 / 80$ & & Control & -6.3 & & \\
\hline \multirow{4}{*}{ Ali et al. [38] } & \multirow{4}{*}{ NA } & \multirow{4}{*}{30} & SE & & & \\
\hline & & & 2\% CHX-ethanol & $64.2^{*}$ & & \\
\hline & & & $2 \% \mathrm{CHX}-\mathrm{dH}_{2} \mathrm{O}$ & $-13.8^{*}$ & & \\
\hline & & & Control & 36.5 & & \\
\hline \multirow{3}{*}{ Leitune et al. [39] } & \multirow{3}{*}{ NA } & \multirow{3}{*}{40} & E\&R & & & \\
\hline & & & CHX $2 \%$ & $-9.8^{*}$ & & \\
\hline & & & Control & 10.9 & & \\
\hline \multirow{3}{*}{ Cova et al. [40] } & Group: immediate $/ 6 \mathrm{~m} / 12 \mathrm{~m}$ & \multirow{3}{*}{60} & E\&R & & & \\
\hline & RF $0.1 \%: 98 / 95 / 97$ & & RF $0.1 \%$ & $19.8^{*}$ & $30.4^{*}$ & \\
\hline & Control: 89/89/95 & & Control & 41.0 & 52.6 & \\
\hline \multirow{5}{*}{ Mobarak [41] } & \multirow{5}{*}{ NA } & & SE & & & $24 \mathrm{~m}$ \\
\hline & & & CHX 2\% & & & ND $63.3 \mathrm{AD}$ \\
\hline & & & & & & 52.1 \\
\hline & & 120 & CHX 5\% & & & $\begin{array}{l}\text { ND } 57.7 \mathrm{AD} \\
28.8^{*}\end{array}$ \\
\hline & & & Control & & & ND $61.1 \mathrm{AD}$ \\
\hline & & & E\&R SB Multipurpose ${ }^{\circledR}$ & & & 3.1 \\
\hline & & & CHX $2 \%$ WUW & & & $9 \mathrm{~m}: 9.518 \mathrm{~m}:$ \\
\hline & & & CHX 2\% WWB & & & 26.1 \\
\hline & & & Control & & & $9 \mathrm{~m}: 15.5$ \\
\hline & & & . 10 & & & $18 \mathrm{~m}: 26.2$ \\
\hline & & & Single Bond $2^{\circledR}$ & & & \\
\hline Sadek et al. [42] & NA & 42 & CHX 2\% WWB & & & $\begin{array}{l}9 \mathrm{~m}: 10.4 \\
18 \mathrm{~m}: 32.4\end{array}$ \\
\hline & & & & & & $9 \mathrm{~m}: 18.9$ \\
\hline & & & Control & & & $18 \mathrm{~m}: 25.7$ \\
\hline & & & Experimental & & & \\
\hline & & & CHX 2\% EWB & & & $\begin{array}{c}9 \mathrm{~m}: 4.718 \mathrm{~m}: \\
7.0\end{array}$ \\
\hline & & & Control & & & $\begin{array}{c}9 \mathrm{~m}: 3.018 \mathrm{~m}: \\
3.3\end{array}$ \\
\hline
\end{tabular}


TABle 1: Continued.

\begin{tabular}{|c|c|c|c|c|c|c|}
\hline \multirow[t]{2}{*}{ Article } & \multirow{2}{*}{$\begin{array}{l}\text { Adhesive/mixed failure modes (\%) in } \\
\text { groups immediately/after aging }\end{array}$} & \multirow[t]{2}{*}{$N$} & \multirow{2}{*}{$\begin{array}{l}\text { Technique of bonding (E\&R/ } \\
\text { SE) MMP inhibitor }+\%\end{array}$} & \multicolumn{3}{|c|}{$\begin{array}{c}\text { Bond strength reduction (\%) } \\
\text { after aging }\end{array}$} \\
\hline & & & & $6 \mathrm{~m}$ & $12 \mathrm{~m}$ & $>12 \mathrm{~m}$ \\
\hline \multirow{8}{*}{ Stanislawczuk et al. [43] } & \multirow{8}{*}{ NA } & \multirow{8}{*}{42} & E\&R Prime \& Bond $\mathrm{NT}^{\circledR}$ & & & $24 \mathrm{~m}$ \\
\hline & & & Control & & & 53.5 \\
\hline & & & CHX & & & $19.2^{*}$ \\
\hline & & & CHX-PA & & & $21.8^{*}$ \\
\hline & & & E\&R Adper Single Bond ${ }^{\circledR}$ & & & \\
\hline & & & Control & & & 46.9 \\
\hline & & & CHX & & & $18.9^{*}$ \\
\hline & & & CHX-PA & & & $16.3^{*}$ \\
\hline \multirow{12}{*}{ De Munck et al. [44] } & $\begin{array}{l}\text { Group: Immediate } / 6 \mathrm{~m} / 12 \mathrm{~m} \\
\text { Scotchbond } 1 \mathrm{XT}^{\circledR}\end{array}$ & \multirow{12}{*}{45} & $\mathrm{E} \& \mathrm{R}$ & & & \\
\hline & Control: 20/70/70 & & Control & 42.9 & 49.5 & \\
\hline & CHX: 63/69/100 & & CHX & 41.4 & 78.9 & \\
\hline & SB-3CT: 33/100/100 & & SB-3CT & 76.5 & 93.6 & \\
\hline & Clearfil Protect Bond ${ }^{\circledR}$ & & SE & & & \\
\hline & Control: 0/60/54 & & Control & 21 & 33.5 & \\
\hline & CHX: 0/63/67 & & CHX & 33.1 & 48.3 & \\
\hline & SB-3CT: $6 / 32 / 82$ & & SB-3CT & 30.4 & 57.5 & \\
\hline & G-Bond ${ }^{\circledR}$ & & SE & & & \\
\hline & Control: 95/95/100 & & Control & 52.1 & 66.2 & \\
\hline & CHX: 90/93/100 & & CHX & 35.3 & 79.1 & \\
\hline & SB-3CT: $89 / 100 / 100$ & & SB-3CT & 62.3 & 60.8 & \\
\hline \multirow{3}{*}{ Ricci et al. [45] } & Group: immediate $/ 10-12 \mathrm{~m} / 18-20 \mathrm{~m}$ & \multirow{3}{*}{26} & E\&R & & $10-12 \mathrm{~m}$ & $18-20 \mathrm{~m}$ \\
\hline & CHX 2\%: 100/100/100 & & CHX $2 \%$ & & 26.3 & $37.0^{*}$ \\
\hline & Control: 75/87.5/100 & & Control & & 43.9 & 56.5 \\
\hline \multirow{3}{*}{ Breschi et al. [46] } & Group: immediate/12 m & \multirow{3}{*}{28} & E\&R & & & \\
\hline & GAL $/ 85 / 75$ & & GAL $0.04 \%$ & & $26.5^{*}$ & \\
\hline & Control: $65 / 76$ & & Control & & 45.4 & \\
\hline \multirow{4}{*}{ Breschi et al. [47] } & Group: immediate $/ 24 \mathrm{~m}$ & \multirow{4}{*}{48} & E\&R & & & $24 \mathrm{~m}$ \\
\hline & Control: 100/80 & & Control & & & 67.2 \\
\hline & CHX 0.2\%: 85/100 & & CHX $0.2 \%$ & & & $16.8^{*}$ \\
\hline & CHX 2\%: 90/75 & & CHX 2\% & & & $30.8^{*}$ \\
\hline \multirow{14}{*}{ Loguercio et al. [48] } & $\begin{array}{l}\text { Group: immediate } / 6 \mathrm{~m} \\
\text { Prime \& Bond } 2.1^{\circledR}\end{array}$ & \multirow{14}{*}{120} & $\mathrm{E} \& \mathrm{R}$ & & & \\
\hline & Control: $75 / 80.9$ & & Control & 33.4 & & \\
\hline & $0.002 \%: 77.6 / 71.7$ & & CHX $0.002 \%$ & 11 & & \\
\hline & $0.02 \%: 67.9 / 75$ & & CHX $0.02 \%$ & $-0.7^{*}$ & & \\
\hline & $0.2 \%: 87.5 / 94.1$ & & CHX $0.2 \%$ & $11.3^{*}$ & & \\
\hline & $2 \%: 75 / 94.4$ & & $\mathrm{CHX} 2 \%$ & $8.5^{*}$ & & \\
\hline & $4 \%: 88.2 / 76$ & & $\mathrm{CHX} 4 \%$ & 21 & & \\
\hline & Adper Single Bond ${ }^{\circledR}$ & & E\&R & & & \\
\hline & Control: $81.2 / 69$ & & Control & 29 & & \\
\hline & $0.002 \%: 73.5 / 76.1$ & & CHX 0.002\% & 11.6 & & \\
\hline & $0.02 \%: 46.4 / 75$ & & CHX $0.02 \%$ & $9.6^{*}$ & & \\
\hline & $0.2 \%: 80 / 88$ & & CHX $0.2 \%$ & $-5.8^{*}$ & & \\
\hline & $2 \%: 84.1 / 97.1$ & & CHX 2\% & $12.7^{*}$ & & \\
\hline & 4\%: $77.6 / 70.2$ & & CHX $4 \%$ & 7.6 & & \\
\hline \multirow{8}{*}{ Stanislawczuk et al. [49] } & $\begin{array}{l}\text { Group: immediate } / 6 \mathrm{~m} \\
\text { Prime \& Bond } \mathrm{NT}^{\circledR}\end{array}$ & & $\mathrm{E} \& \mathrm{R}$ & & & \\
\hline & Control: $67 / 75.8$ & & Control & 33.6 & & \\
\hline & CHX 2\%: 75/96.5 & & CHX $2 \%$ & $-6.8^{*}$ & & \\
\hline & CHX 2\%-PA: 83/72.4 & 42 & CHX 2\%-PA & $16^{*}$ & & \\
\hline & Single Bond $2^{\circledR}$ & & E\&R & & & \\
\hline & Control: $85 / 64.3$ & & Control & 25 & & \\
\hline & CHX 2\%: 96.3/100 & & CHX $2 \%$ & $0^{*}$ & & \\
\hline & CHX 2\%-PA: 87.6/76.9 & & CHX 2\%-PA & $4.6^{*}$ & & \\
\hline
\end{tabular}


Table 1: Continued.

\begin{tabular}{|c|c|c|c|c|c|c|}
\hline \multirow[t]{2}{*}{ Article } & \multirow{2}{*}{$\begin{array}{l}\text { Adhesive/mixed failure modes (\%) in } \\
\text { groups immediately/after aging }\end{array}$} & \multirow[t]{2}{*}{$N$} & \multirow{2}{*}{$\begin{array}{l}\text { Technique of bonding (E\&R/ } \\
\text { SE) MMP inhibitor }+\%\end{array}$} & \multicolumn{3}{|c|}{$\begin{array}{c}\text { Bond strength reduction (\%) } \\
\text { after aging }\end{array}$} \\
\hline & & & & $6 \mathrm{~m}$ & $12 \mathrm{~m}$ & $>12 \mathrm{~m}$ \\
\hline \multirow{9}{*}{ Zhou et al. [50] } & Group: immediate $/ 12 \mathrm{~m}$ & \multirow{9}{*}{16} & SE & & & \\
\hline & 0.05\% CHX: 93.8/100 & & 0.05\% CHX & & 18.1 & \\
\hline & Control: $87.5 / 93.8$ & & Control & & 18.7 & \\
\hline & 0.1\% CHX: 75/93.8 & & $0.1 \% \mathrm{CHX}$ & & $-0.8^{*}$ & \\
\hline & Control: 93.8/100 & & Control & & 16.0 & \\
\hline & 0.5\% CHX: 87.5/100 & & $0.5 \% \mathrm{CHX}$ & & $5.9^{*}$ & \\
\hline & Control: 93.8/100 & & Control & & 21.8 & \\
\hline & $1 \% \mathrm{CHX} / 93.8 / 93.8$ & & $1 \% \mathrm{CHX}$ & & $2.9^{*}$ & \\
\hline & Control: 87.5/100 & & Control & & 15.6 & \\
\hline \multirow{7}{*}{ Breschi et al. [51] } & $\begin{array}{l}\text { Group: immediate } / 6 \mathrm{~m} / 12 \mathrm{~m} \\
\text { Single Bond } 1 \mathrm{XT}^{\circledR}\end{array}$ & \multirow{7}{*}{108} & $\mathrm{E} \& \mathrm{R}$ & & & \\
\hline & CHX 2\%: 90/95/95 & & $\mathrm{CHX} 2 \%$ & 11 & $24.6^{*}$ & \\
\hline & CHX 0.2\%: $100 / 95 / 100$ & & CHX $0.2 \%$ & 16.5 & $20.8^{*}$ & \\
\hline & $\begin{array}{c}\text { Control: } 100 / 90 / 90 \\
\text { XP-Bond }{ }^{\circledR}\end{array}$ & & $\begin{array}{l}\text { Control } \\
\text { E\&R }\end{array}$ & 38.0 & 54.2 & \\
\hline & CHX 2\%: 100/90/85 & & $\mathrm{CHX} 2 \%$ & 14.4 & $24.2^{*}$ & \\
\hline & CHX 0.2\%: 95/100/90 & & CHX $0.2 \%$ & 13.1 & $30.8^{*}$ & \\
\hline & Control: 100/95/85 & & Control & 33.1 & 64.1 & \\
\hline
\end{tabular}

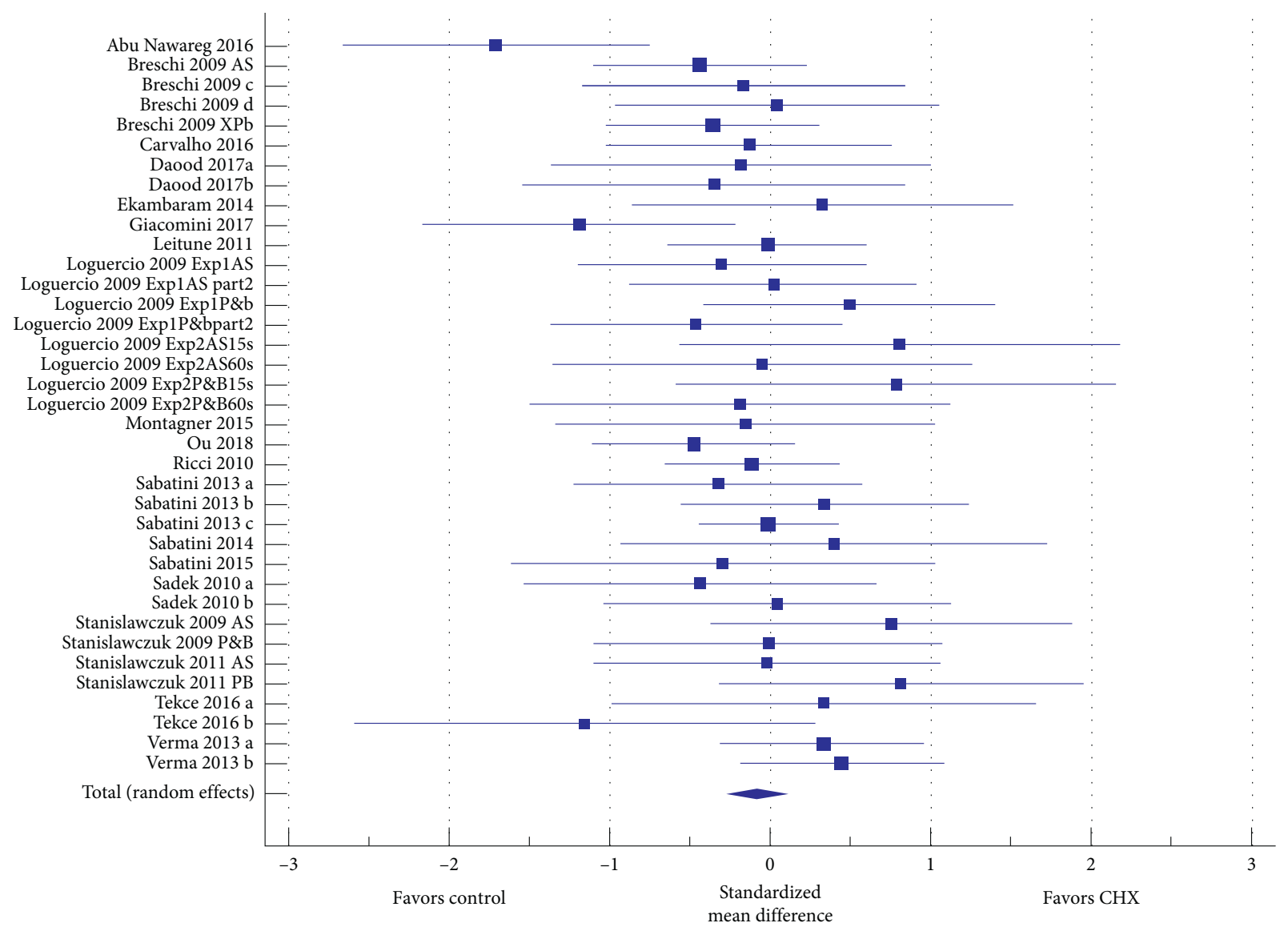

Figure 2: Forest plot of studies at baseline. The $N$ for both groups was 396 samples. The total random effect standardized mean difference (SMD) was -0.0821 (CI 95\%-0.240; 0.076). The difference was not statistically significant $(t=-1.019, P=0.308)$. The $I^{2}$ (inconsistency) was $21.68 \%$. 


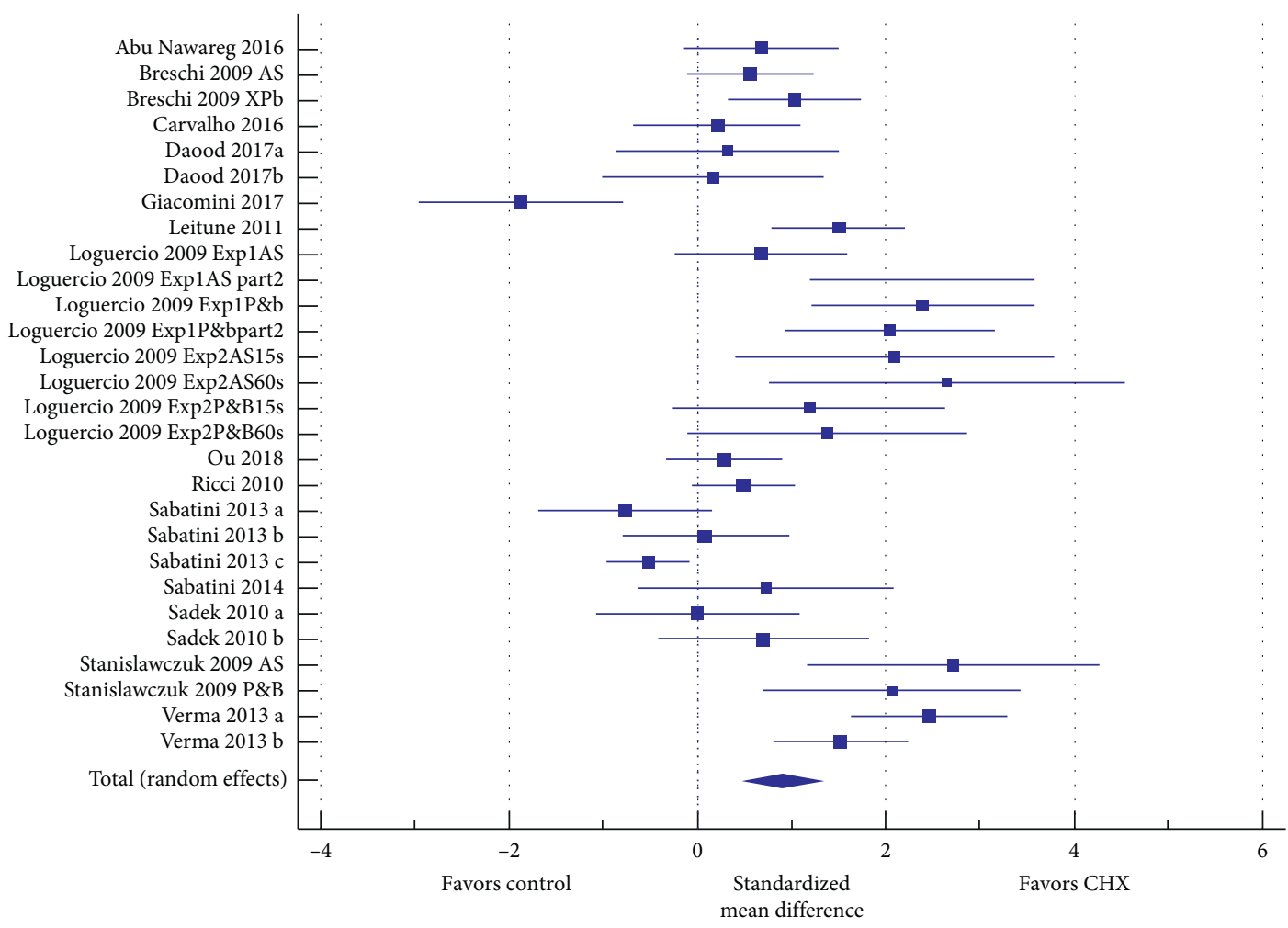

(a)

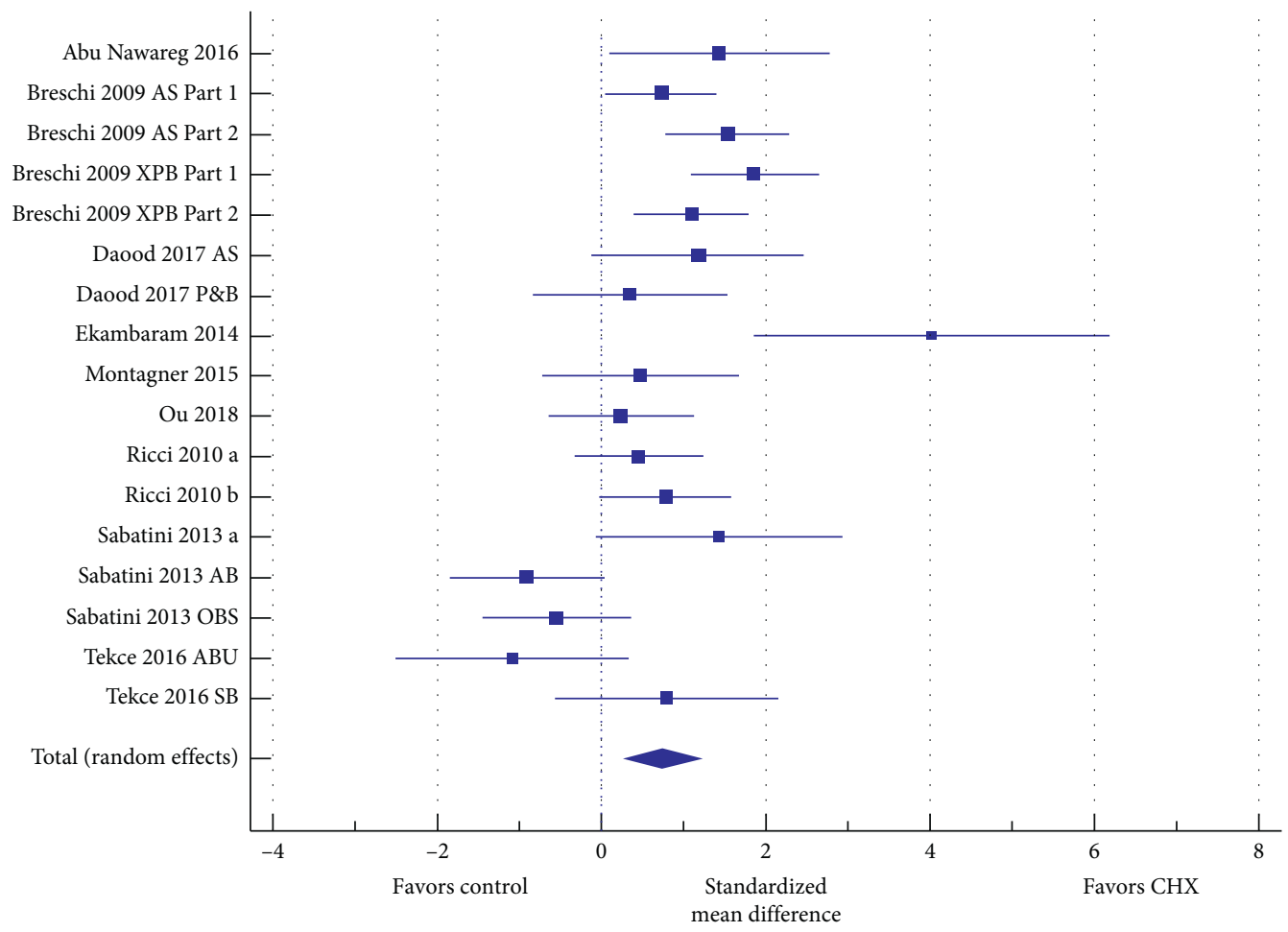

(b)

Figure 3: Continued. 


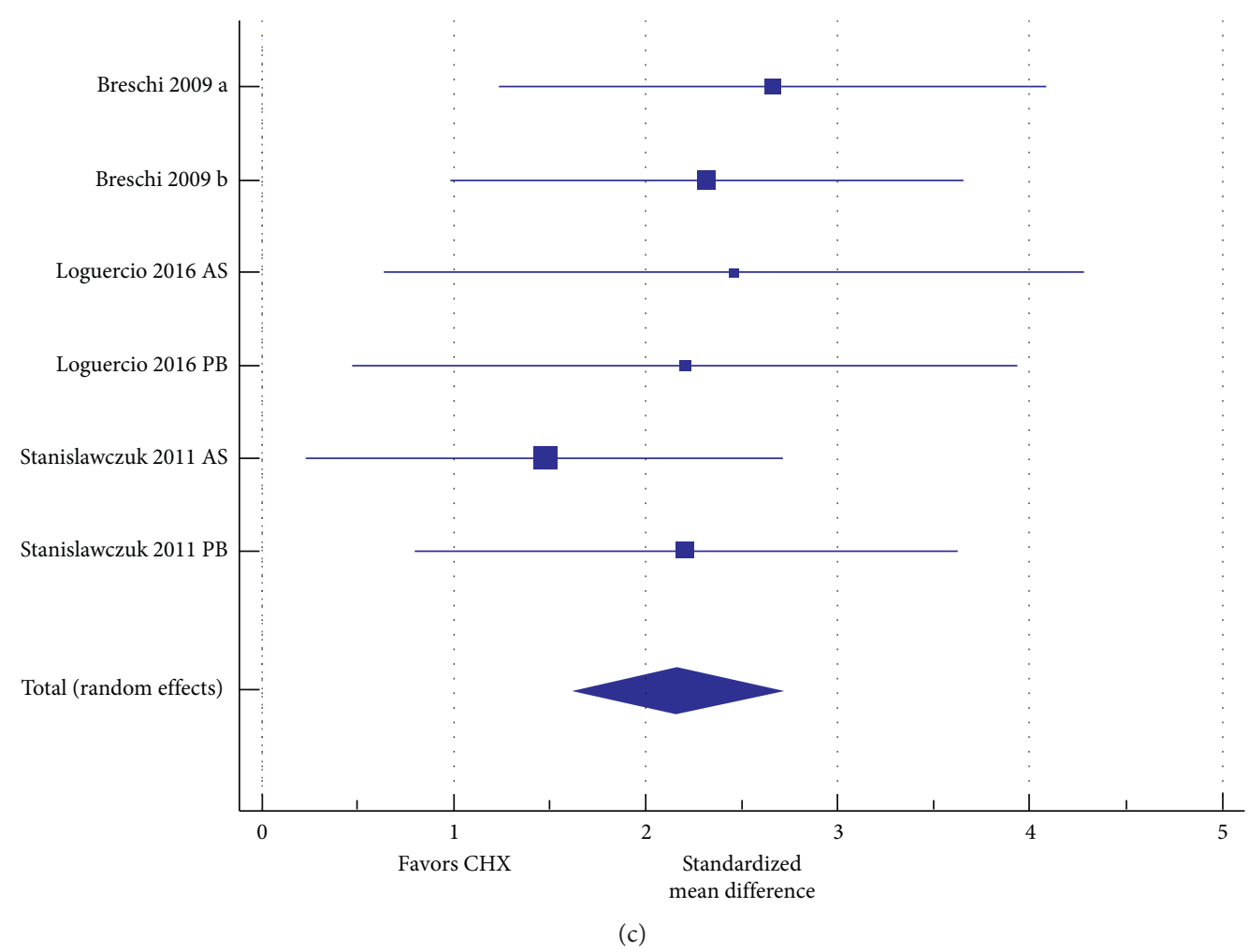

Figure 3: Forest plots of studies after 6, 12, and 24 months of aging. (a) Chlorhexidine (CHX) vs. control at six months. The $N$ for both groups was 339 samples. The total random effect SMD was 0.907 (CI 95\% 0.517; 1.297). The difference was statistically significant $(t=4.568$, $P<0.001$ ). The $I^{2}$ was $82.11 \%$. (b) CHX vs. control after 12 month aging. The $N$ for both groups was 173 samples. The total random effect SMD was 0.821 (CI 95\% 0.367; 1.275). The difference was statistically significant $\left(t=3.557, P<0.001\right.$ ). The $I^{2}$ was $74.97 \%$. (c) CHX vs. control after 24-month aging. The $N$ for both groups was 40 samples. The total random effect SMD was 2.168 (CI 95\% 1.627; 2.708). The difference was statistically significant $(t=7.982, P<0.001)$. The $I^{2}$ was $0.00 \%$.

TABLE 2: Factors associated with the risk of bias in different studies.

\begin{tabular}{|c|c|c|c|c|c|c|c|}
\hline Study & Materials & Caries & Adhesive & Sample & Blinding & Random & Risk \\
\hline Ou et al. [11] & $\mathrm{Y}$ & $\mathrm{Y}$ & NM & NM & NM & $\mathrm{Y}$ & Medium \\
\hline Giacomini et al. [14] & $\mathrm{Y}$ & $\mathrm{Y}$ & NM & NM & NM & NM & High \\
\hline Daood et al. [15] & $\mathrm{Y}$ & $\mathrm{Y}$ & NM & NM & NM & $\mathrm{Y}$ & Medium \\
\hline Carvalho et al. [17] & NM & $\mathrm{N}$ & $\mathrm{Y}$ & $\mathrm{NM}$ & NM & $\mathrm{Y}$ & High \\
\hline Abu Nawareg et al. [23] & $\mathrm{Y}$ & $\mathrm{Y}$ & NM & NM & NM & $\mathrm{Y}$ & Medium \\
\hline Loguercio et al. [20] & $\mathrm{Y}$ & $\mathrm{Y}$ & $\mathrm{Y}$ & NM & NM & $\mathrm{Y}$ & Medium \\
\hline Tekçe et al. [22] & $\mathrm{Y}$ & $\mathrm{Y}$ & NM & NM & NM & $\mathrm{Y}$ & Medium \\
\hline Montagner et al. [25] & $\mathrm{Y}$ & $\mathrm{Y}$ & $\mathrm{Y}$ & $\mathrm{NM}$ & $\mathrm{NM}$ & $\mathrm{Y}$ & Medium \\
\hline Ekambaram et al. [30] & NM & $\mathrm{Y}$ & NM & NM & NM & $\mathrm{Y}$ & High \\
\hline Sabatini et al. [31] & $\mathrm{Y}$ & $\mathrm{Y}$ & NM & NM & NM & $\mathrm{Y}$ & Medium \\
\hline Verma et al. [33] & NM & $\mathrm{Y}$ & NM & NM & NM & $\mathrm{Y}$ & High \\
\hline Sabatini et al. [35] & $\mathrm{Y}$ & $\mathrm{Y}$ & NM & NM & NM & Y & Medium \\
\hline Sabatini [37] & $\mathrm{Y}$ & $\mathrm{Y}$ & NM & NM & NM & $\mathrm{Y}$ & Medium \\
\hline Leitune et al. [39] & $\mathrm{Y}$ & $\mathrm{Y}$ & NM & NM & NM & $\mathrm{Y}$ & Medium \\
\hline Stanislawczuk et al. [43] & NM & $\mathrm{Y}$ & $\mathrm{Y}$ & NM & NM & NM & High \\
\hline Sadek et al. [42] & $\mathrm{Y}$ & $\mathrm{Y}$ & NM & NM & NM & $\mathrm{Y}$ & Medium \\
\hline Ricci et al. [45] & $\mathrm{Y}$ & $N$ & $\mathrm{Y}$ & NM & NM & $\mathrm{Y}$ & Medium \\
\hline Loguercio et al. [48] & $\mathrm{Y}$ & $\mathrm{Y}$ & $\mathrm{Y}$ & NM & NM & $\mathrm{Y}$ & Medium \\
\hline Stanislawczuk et al. [49] & NM & $\mathrm{Y}$ & $\mathrm{Y}$ & NM & NM & NM & High \\
\hline Breschi et al. et al. [51] & $\mathrm{Y}$ & $\mathrm{Y}$ & NM & NM & NM & $\mathrm{Y}$ & Medium \\
\hline Loguercio et al. [48] & $\mathrm{Y}$ & $\mathrm{Y}$ & $\mathrm{Y}$ & NM & NM & $\mathrm{Y}$ & Medium \\
\hline Total 21 & 16 & 19 & 8 & 0 & 0 & 18 & \\
\hline
\end{tabular}


both $[2,6]$. This supports the idea that bond strength decreases over time and that the adhesive interface plays a significant role in the mode of fracture.

A thorough risk of bias assessment was also carried out to identify the main factors which could affect the creditability of the findings. Five articles were classified to have a high risk of bias, and none with low risk. The results are in line with the respective previous study [8]. None of the studies mentioned sample size calculations, and all but one failed to mention the blinding of the operator performing the bond strength testing. The results may reflect the standard level of reporting of bond strength studies, but at least the blinding of the person performing the bond strength testing should be done and also reported.

\section{Conclusions}

This systematic review and meta-analysis demonstrated that studies strongly indicate the benefits of collagen-degrading enzyme inhibition on the preservation of dentin bond strength. Since CHX does not have any adverse effects on the immediate bond strength, the clinical use of CHX can be recommended to increase the longevity of resin-dentin bonds.

\section{Data Availability}

The data used to support the findings of this study are available from the corresponding author upon request.

\section{Conflicts of Interest}

The authors declare that they have no conflicts of interest.

\section{Authors' Contributions}

Kiuru O. and Sinervo J, the first two authors, had an equal contribution to the article.

\section{References}

[1] M. Hashimoto, H. Ohno, M. Kaga, K. Endo, H. Sano, and H. Oguchi, "In vivo degradation of resin-dentin bonds in humans over 1 to 3 years," Journal of Dental Research, vol. 79, no. 6, pp. 1385-1391, 2000.

[2] L. Tjäderhane, F. D. Nascimento, L. Breschi et al., "Optimizing dentin bond durability: control of collagen degradation by matrix metalloproteinases and cysteine cathepsins," Dental Materials, vol. 29, no. 1, pp. 116-135, 2013.

[3] D. H. Pashley, F. R. Tay, L. Breschi et al., "State of the art etchand-rinse adhesives," Dental Materials, vol. 27, no. 1, pp. 1-16, 2011.

[4] B. Van Meerbeek, K. Yoshihara, Y. Yoshida, A. Mine, J. De Munck, and K. L. Van Landuyt, "State of the art of selfetch adhesives," Dental Materials, vol. 27, no. 1, pp. 17-28, 2011.

[5] F. M. Collares, S. B. Rodrigues, V. C. Leitune, R. K. Celeste, F. Borba de Araújo, and S. M. Samuel, "Chlorhexidine application in adhesive procedures: a meta-regression analysis," The Journal of Adhesive Dentistry, vol. 15, no. 1, pp. 11-18, 2013.
[6] A. Mazzoni, L. Tjäderhane, V. Checchi et al., "Role of dentin MMPs in caries progression and bond stability," Journal of Dental Research, vol. 94, no. 2, pp. 241-251, 2015.

[7] A. K. Bedran-Russo, G. F. Pauli, S.-N. Chen et al., "Dentin biomodification: strategies, renewable resources and clinical applications," Dental Materials, vol. 30, no. 1, pp. 62-76, 2014.

[8] A. F. Montagner, R. Sarkis-Onofre, T. Pereira-Cenci, and M. S. Cenci, "MMP inhibitors on dentin stability," Journal of Dental Research, vol. 93, no. 8, pp. 733-743, 2014.

[9] J. Li, B. Chen, N. Hong, S. Wu, and Y. Li, "Effect of baicalein on matrix metalloproteinases and durability of resin-dentin bonding," Operative Dentistry, vol. 43, no. 4, pp. 426-436, 2018.

[10] P. Malaquias, M. Gutierrez, V. Hass et al., "Two year effects of chlorhexidine-containing adhesives on the in vitro durability of resin-dentin interfaces and modeling of drug release," Operative Dentistry, vol. 43, no. 2, pp. 201-212, 2018.

[11] Q. Ou, Y. Hu, S. Yao, Y. Wang, and X. Lin, "Effect of matrix metalloproteinase 8 inhibitor on resin-dentin bonds," Dental Materials, vol. 34, no. 5, pp. 756-763, 2018.

[12] M. El Gezawi, R. Haridy, E. Abo Elazm, F. Al-Harbi, M. Zouch, and D. Kaisarly, "Microtensile bond strength, 4point bending and nanoleakage of resin-dentin interfaces: effects of two matrix metalloproteinase inhibitors," Journal of the Mechanical Behavior of Biomedical Materials, vol. 78, pp. 206-213, 2018.

[13] T. Maravic, L. Breschi, A. Comba et al., "Experimental use of an acrolein-based primer as collagen cross-linker for dentine bonding," Journal of Dentistry, vol. 68, pp. 85-90, 2018.

[14] M. Giacomini, P. Scaffa, L. Chaves et al., "Role of proteolytic enzyme inhibitors on carious and eroded dentin associated with a universal bonding system," Operative Dentistry, vol. 42, no. 6, pp. E188-E196, 2017.

[15] D. Daood, C. K. Y. Yiu, M. F. Burrow, L.-N. Niu, and F. R. Tay, "Effect of a novel quaternary ammonium silane cavity disinfectant on durability of resin-dentine bond," Journal of Dentistry, vol. 60, pp. 77-86, 2017.

[16] B. Venigalla, P. Jyothi, S. Kamishetty, S. Reddy, R. Cherukupalli, and D. Reddy, "Resin bond strength to water versus ethanol-saturated human dentin pretreated with three different cross-linking agents," Journal of Conservative Dentistry, vol. 19, no. 6, pp. 555-559, 2016.

[17] C. Carvalho, F. P. Fernandes, V. d. P. Freitas et al., "Effect of green tea extract on bonding durability of an etch-and-rinse adhesive system to caries-affected dentin," Journal of Applied Oral Science, vol. 24, no. 3, pp. 211-217, 2016.

[18] D. C. Barcellos, B. M. Fonseca, C. R. Pucci, B. d. N. Cavalcanti, E. D. S. Persici, and S. E. d. P. Gonçalves, "Zn-doped etch-andrinse model dentin adhesives: dentin bond integrity, biocompatibility, and properties," Dental Materials, vol. 32, no. 7, pp. 940-950, 2016.

[19] V. Hass, I. V. Luque-Martinez, M. F. Gutierrez et al., "Collagen cross-linkers on dentin bonding: stability of the adhesive interfaces, degree of conversion of the adhesive, cytotoxicity and in situ MMP inhibition," Dental Materials, vol. 32, no. 6, pp. 732-741, 2016.

[20] A. Loguercio, R. Stanislawczuk, P. Malaquias, M. Gutierrez, J. Bauer, and A. Reis, "Effect of minocycline on the durability of dentin bonding produced with etch-and-rinse adhesives," Operative Dentistry, vol. 41, no. 5, pp. 511-519, 2016.

[21] V. Hass, I. Luque-Martinez, M. A. Muñoz et al., "The effect of proanthocyanidin-containing $10 \%$ phosphoric acid on bonding properties and," MMP Inhibition Dental Materials, vol. 32, no. 3, pp. 468-475, 2016. 
[22] N. Tekçe, S. Tuncer, M. Demirci, and S. Balci, "Do matrix metalloproteinase inhibitors improve the bond durability of universal dental adhesives?" Scanning, vol. 38, no. 6, pp. 535-544, 2016.

[23] M. Abu Nawareg, D. Elkassas, A. Zidan et al., "Is chlorhexidine-methacrylate as effective as chlorhexidine digluconate in preserving resin dentin interfaces?" Journal of Dentistry, vol. 45 , pp. 7-13, 2016.

[24] E. M. da Silva, C. U. F. de Sá Rodrigues, M. P. de Oliveira Matos, T. R. de Carvalho, G. B. dos Santos, and C. M. Amaral, "Experimental etch-and-rinse adhesive systems containing MMP-inhibitors: physicochemical characterization and resin-dentin bonding stability," Journal of Dentistry, vol. 43, no. 12, pp. 1491-1497, 2015.

[25] A. F. Montagner, T. Pereira-Cenci, and M. S. Cenci, "Influence of cariogenic challenge on bond strength stability of dentin," Brazilian Dental Journal, vol. 26, no. 2, pp. 128-134, 2015.

[26] C. Sabatini, P. A. Ortiz, and D. H. Pashley, "Preservation of resin-dentin interfaces treated with benzalkonium chloride adhesive blends," European Journal of Oral Sciences, vol. 123, no. 2, pp. 108-115, 2015.

[27] C. Sabatini and D. H. Pashley, "Aging of adhesive interfaces treated with benzalkonium chloride and benzalkonium methacrylate," European Journal of Oral Sciences, vol. 123, no. 2, pp. 102-107, 2015.

[28] C. B. André, B. P. F. A. Gomes, T. M. Duque et al., "Dentine bond strength and antimicrobial activity evaluation of adhesive systems," Journal of Dentistry, vol. 43, no. 4, pp. 466-475, 2015.

[29] A. P. Manso, R. H. Grande, A. K. Bedran-Russo et al., "Can 1\% chlorhexidine diacetate and ethanol stabilize resin-dentin bonds?” Dental Materials, vol. 30, no. 7, pp. 735-741, 2014.

[30] M. Ekambaram, C. K. Y. Yiu, J. P. Matinlinna, N. M. King, and F. R. Tay, "Adjunctive application of chlorhexidine and ethanol-wet bonding on durability of bonds to sound and caries-affected dentine," Journal of Dentistry, vol. 42, no. 6, pp. 709-719, 2014.

[31] C. Sabatini and S. K. Patel, "Matrix metalloproteinase inhibitory properties of benzalkonium chloride stabilizes adhesive interfaces," European Journal of Oral Sciences, vol. 121, no. 6, pp. 610-616, 2013.

[32] C. Pomacóndor-Hernández, A. N. d. G. Antunes, V. d. Hipólito, and M. F. d. Goes, "Effect of replacing a component of a self-etch adhesive by chlorhexidine on bonding to dentin," Brazilian Dental Journal, vol. 24, no. 4, pp. 335-339, 2013.

[33] R. Verma, U. P. Singh, S. P. Tyagi, R. Nagpal, and N. Manuja, "Long-term bonding effectiveness of simplified etch-andrinse adhesives to dentin after different surface pre-treatments," Journal of Conservative Dentistry: JCD, vol. 16, no. 4, pp. 367-370, 2013.

[34] L. Tjäderhane, P. Mehtälä, P. Scaffa et al., "The effect of dimethyl sulfoxide (DMSO) on dentin bonding and nanoleakage of etch-and-rinse adhesives," Dental Materials, vol. 29, no. 10, pp. 1055-1062, 2013.

[35] C. Sabatini, J. Kim, and P. O. Alias, "In vitro evaluation of benzalkonium chloride in the preservation of adhesive interfaces," Operative Dentistry, vol. 39, no. 3, pp. 283-290, 2014.

[36] D. Simões, R. Basting, F. Amaral, C. Turssi, and F. França, "Influence of chlorhexidine and/or ethanol treatment on bond strength of an etch-and-rinse adhesive to dentin: an in vitro and in situ study," Operative Dentistry, vol. 39, no. 1, pp. 64-71, 2014.

[37] C. Sabatini, "Effect of a chlorhexidine-containing adhesive on dentin bond strength stability," Operative Dentistry, vol. 38, no. 6, pp. 609-617, 2013.

[38] A. Ali, H. El Deeb, O. Badran, and E. Mobarak, "Bond durability of self-etch adhesive to ethanol-based chlorhexidine pretreated dentin after storage in artificial saliva and under intrapulpal pressure simulation," Operative Dentistry, vol. 38, no. 4, pp. 439-446, 2013.

[39] V. C. B. Leitune, F. F. Portella, P. V. Bohn, F. M. Collares, and S. M. W. Samuel, "Influence of chlorhexidine application on longitudinal adhesive bond strength in deciduous teeth," Brazilian Oral Research, vol. 25, no. 5, pp. 388-392, 2011.

[40] A. Cova, L. Breschi, F. Nato et al., "Effect of UVA-activated riboflavin on dentin bonding," Journal of Dental Research, vol. 90, no. 12, pp. 1439-1445, 2011.

[41] E. Mobarak, "Effect of chlorhexidine pretreatment on bond strength durability of caries-affected dentin over 2 year aging in artificial saliva and under simulated intrapulpal pressure," Operative Dentistry, vol. 36, no. 6, pp. 649-660, 2011.

[42] F. T. Sadek, R. R. Braga, A. Muench, Y. Liu, D. H. Pashley, and F. R. Tay, "Ethanol wet-bonding challenges current antidegradation strategy," Journal of Dental Research, vol. 89, no. 12, pp. 1499-1504, 2010.

[43] R. Stanislawczuk, A. Reis, and A. D. Loguercio, “A 2 year in vitro evaluation of a chlorhexidine-containing acid on the durability of resin-dentin interfaces," Journal of Dentistry, vol. 39, no. 1, pp. 40-47, 2011.

[44] J. De Munck, A. Mine, P. E. Van den Steen et al., "Enzymatic degradation of adhesive-dentin interfaces produced by mild self-etch adhesives," European Journal of Oral Sciences, vol. 118, no. 5, pp. 494-501, 2010.

[45] H. A. Ricci, M. E. Sanabe, C. A. de Souza Costa, D. H. Pashley, and J. Hebling, "Chlorhexidine increases the longevity of in vivo resin-dentin bonds," European Journal of Oral Sciences, vol. 118, no. 4, pp. 411-416, 2010.

[46] L. Breschi, P. Martin, A. Mazzoni et al., "Use of a specific MMP-inhibitor (galardin) for preservation of hybrid layer," Dental Materials, vol. 26, no. 6, pp. 571-578, 2010.

[47] L. Breschi, A. Mazzoni, F. Nato et al., "Chlorhexidine stabilizes the adhesive interface: a 2 year in vitro study," Dental Materials, vol. 26, no. 4, pp. 320-325, 2010.

[48] A. D. Loguercio, R. Stanislawczuk, L. G. Polli, J. A. Costa, M. D. Michel, and A. Reis, "Influence of chlorhexidine digluconate concentration and application time on resindentin bond strength durability," European Journal of Oral Sciences, vol. 117, no. 5, pp. 587-596, 2009.

[49] R. Stanislawczuk, R. C. Amaral, C. Zander-Grande, D. Gagler, A. Reis, and A. D. Loguercio, "Chlorhexidine-containing acid conditioner preserves the longevity of resin-dentin bonds," Operative Dentistry, vol. 34, no. 4, pp. 481-490, 2009.

[50] J. Zhou, J. Tan, L. Chen, D. Li, and Y. Tan, "The incorporation of chlorhexidine in a two-step self-etching adhesive preserves dentin bond in vitro," Journal of Dentistry, vol. 37, no. 10, pp. 807-812, 2009.

[51] L. Breschi, F. Cammelli, E. Visintini et al., "Influence of chlorhexidine concentration on the durability of etch-andrinse dentin bonds: a 12 month in vitro study," The Journal of Adhesive Dentistry, vol. 11, no. 3, pp. 191-198, 2009. 